\title{
Kernos
}

Revue internationale et pluridisciplinaire de religion grecque antique

$25 \mid 2012$

Varia

\section{Tommaso ISMAELLI, Archeologia del culto a Gela. Il santuario del Predio Sola}

Nicola Cucuzza

OpenEdition

Journals

\section{Edizione digitale}

URL: http://journals.openedition.org/kernos/2067

DOI: $10.4000 /$ kernos.2067

ISSN: 2034-7871

\section{Editore}

Centre international d'étude de la religion grecque antique

\section{Edizione cartacea}

Data di pubblicazione: 26 octobre 2012

Paginazione: 362-365

ISSN: 0776-3824

\section{Notizia bibliografica digitale}

Nicola Cucuzza, «Tommaso ismaelu, Archeologia del culto a Gela. II santuario del Predio Sola », Kernos [En ligne], 25 | 2012, mis en ligne le 01 octobre 2012, consulté le 21 septembre 2020. URL : http:// journals.openedition.org/kernos/2067 ; DOI : https://doi.org/10.4000/kernos.2067

Questo documento è stato generato automaticamente il 21 septembre 2020.

Kernos 


\title{
Tommaso ISMAELLI, Archeologia del culto a Gela. Il santuario del Predio Sola
}

\author{
Nicola Cucuzza
}

\section{NOTIZIA}

Tommaso ISMAelli, Archeologia del culto a Gela. Il santuario del Predio Sola, Bari, Edipuglia, 2011. 1 vol. $21 \times 29,7$ cm, 266 p., 39 fig. +38 tav. (Beni Archeologici - Conoscenza e

Tecnologie. Quaderno, 9). ISBN : 978-88-7228-634-0.

1 La tumultuosa attività edilizia che si sviluppò a Gela negli anni Cinquanta trasformò in pochi anni il piccolo borgo, fino al 1927 noto con il toponimo di Terranova (fondato, dopo un lunghissimo periodo di abbandono, da Federico II nel XIII secolo sul sito della colonia rodio-cretese), nell'odierno agglomerato popolato da oltre centomila persone. Il miraggio di un'occupazione stabile presso il polo petrolchimico creò infatti un vorticoso fenomeno di inurbamento che portò in breve tempo migliaia di famiglie di contadini dell'area dei 'campi geloi' a trasferirsi a Gela. Tale situazione comportò la necessità di costruire in fretta alloggi e strade, ampliando di molto - e molto rapidamente - il moderno abitato di Gela, che si sovrapponeva quasi perfettamente a quello antico, fino ad allora solo in parte esplorato da P. Orsi ed ancor prima, sul finire del XIX secolo, vera e propria fonte di approvvigionamento per alcune collezioni museali europee ${ }^{1}$. L'intensa opera di urbanizzazione, non sempre felice nei risultati ${ }^{2}$, si trasformò, grazie 'all'azione pionieristica' (come ricorda F. D'Andria nella Presentazione, a p.9) ed all'impegno di P.Orlandini, D. Adamesteanu e P.Griffo, ispettori della Soprintendenza di Agrigento, nella possibilità di acquisire parecchi nuovi elementi di conoscenza sull'abitato antico, permettendo anche di avviare su nuove basi metodologiche delle ricerche mirate a comprendere il rapporto fra Gela ed il territorio circostante. I risultati di quelle esplorazioni, che portarono alla creazione del locale 
Museo Archeologico, sono in alcune pubblicazioni che costituiscono tuttora il nucleo principale di conoscenze sull'antica Gela, anche se il prosieguo delle ricerche tende come è logico - a precisare o addirittura a correggere alcune delle conclusioni allora raggiunte.

2 È in quest'ottica che va inquadrato il lavoro di T. Ismaelli (T.I.), che ha riesaminato il materiale recuperato nello scavo del predio Sola, effettuato nella primavera del 1959 e reso noto appena tre anni dopo da una corposa pubblicazione di P. Orlandini ${ }^{3}$. I materiali, portati alla luce in pochi giorni di scavo, furono subito interpretati come pertinenti ad un santuario in uso dalla metà del VII al V secolo a.C., con due distinti livelli (definiti strati), al secondo dei quali appartenevano i resti di una piccola costruzione. Ubicato sul versante meridionale della collina su cui si sviluppava l'abitato, appena ad Ovest del luogo di rinvenimento del frammento di kylix con dedica all'ecista Antifemo, quello del predio Sola venne interpretato come uno dei santuari extra-urbani della polis, al pari di altri sacelli indagati nello stesso periodo (da quello di via Fiume a quello di Madonna dell'Alemanna fino a quello di Bitalemi, già in parte scavato da Orsi) ed allo stesso modo attribuito al culto demetriaco. Lo studio di T.I. cerca di precisare meglio le caratteristiche del culto tributato nel santuario del predio Sola partendo da un esame sistematico di tutti i materiali recuperati nello scavo. L'opera può essere divisa sostanzialmente in tre parti: ad una breve trattazione dedicata all'inquadramento del santuario nella topografia del sito ed al riepilogo dei dati di scavo già presentati da Orlandini (p. 13-24) segue infatti il catalogo e lo studio dei materiali divisi per classi (p. 25-205). L'ultima parte (p. 207-235) si occupa degli aspetti legati al culto, con il tentativo di comprendere le caratteristiche della divinità venerata nel santuario; chiude un'ampia ed accurata bibliografia (p. 237-266). Il volume, che prende spunto dalla tesi di laurea discussa dall'A. presso l'Università di Perugia, costituisce un utile completamento all'articolo di Orlandini, apparso quasi mezzo secolo prima. Rispetto a quella pubblicazione T.I. offre un catalogo completo di tutti i materiali rinvenuti nel corso dello scavo ed attualmente conservati nel locale Museo Archeologico di Gela (ad eccezione di un piccolo gruppo custodito ad Agrigento). L'analisi complessiva dei reperti e le più recenti riflessioni sull'archeologia cultuale permettono all'A. di giungere a conclusioni leggermente diverse da quelle di Orlandini per quanto riguarda sia la cronologia che le attività di culto.

3 Nelle prime pagine T.I. (che pubblica a p. 15 una interessante foto aerea del 1938) lamenta giustamente l'assenza, per Gela, di una vera carta archeologica, che costituirebbe un fondamentale strumento di lavoro e, nello specifico, permetterebbe di definire meglio il carattere del santuario di Predio Sola su un piano topografico. T.I. solleva dei legittimi dubbi sulla classificazione del santuario come extra-urbano: mentre non è stato rinvenuto alcun circuito murario sul lato meridionale della collina, il complesso del Bosco Littorio, a breve distanza dalla riva del mare, e la probabile ubicazione dell'heroon di Antifemo potrebbero testimoniare come le pendici meridionali dell'altura su cui sorgeva Gela fossero urbanizzate. La presenza di diversi terrazzamenti, visibili nelle foto aeree presentate, con dei veri e propri speroni (come quello su cui venne effettuato lo $\mathrm{scavo}^{4}$ ) indica peraltro un'articolazione abbastanza complessa che meriterebbe una attenzione maggiore rispetto a quella che ha finora ricevuto.

4 Anche a causa della ristrettezza dell'area scavata (circa $\mathrm{m} 6 \times 4$ ) non sono ben chiare le caratteristiche dello strato I, dal quale proviene la maggior parte dei reperti. T.I. rifiuta 
la definizione di stipe, data da Orlandini, ma non indica una alternativa (p. 24): forse sarebbe preferibile utilizzare quella di 'deposito votivo', già impiegata nello specifico da Orlandini ${ }^{5}$. La documentazione disponibile sembrerebbe indicare come lo strato I (i cui materiali erano evidentemente in giacitura secondaria) fosse il risultato di uno spianamento per mettere in opera l'unica struttura architettonica portata alla luce. Di quest'ultima si conservano solo due setti murari ortogonali fra loro (dimensioni massime $\mathrm{m} 4,60 \times 2,30$ ); la sua interpretazione come edificio di culto non riposa su dati oggettivi, come del resto anche quella degli altri edifici geloi citati a confronto (strutture di via Fiume, dell'ex-scalo ferroviario etc.). Qualcosa di più potrebbe forse ricavarsi per i materiali dello strato II, che sembrano relativi al momento di uso di questa struttura fino al suo collasso, come lascia sospettare la presenza di coppi, relativi con ogni probabilità alla copertura dello stesso edificio. Dato che Orlandini osservava come almeno una quarantina delle 52 protomi fosse stata trovata lungo $\mathrm{i}$ muri ed in particolare nell'angolo supersite dell'edificio, è forse lecito ritenere che almeno in questo punto si fosse conservato il crollo della struttura e che quindi le protomi fossero probabilmente appese alle pareti: se questa ipotesi fosse corretta, si avrebbe probabilmente un elemento in più per chiedersi se l'edificio vada interpretato come un sacello. Un esame sullo stato di frammentazione dei materiali recuperati avrebbe comunque forse permesso di acquisire dei dati utili a definire meglio il carattere dei due depositi votivi.

Il catalogo e lo studio dei materiali costituiscono la parte più corposa dell'opera. Sono elencati 756 oggetti (o gruppi di oggetti), dei quali 752 pertinenti alla frequentazione cultuale; T.I. segnala che si tratta dell'intero gruppo di oggetti conservati, fatta eccezione per una diecina di frammenti ceramici non classificabili per il precario stato di conservazione (p. 25). Non esclude tuttavia la possibilità che il lotto oggi esistente sia frutto di una selezione operata dagli scavatori: la presenza di due frammenti ceramici preistorici e di due medievali (cat. 753-756, p. 205) alimenta questo sospetto, indicando peraltro come la situazione stratigrafica fosse probabilmente più articolata di quella resa nota. Va in particolare ricordato come i due frammenti preistorici, della fase di Castelluccio, costituiscano un utile dato per l'occupazione preistorica di Gela, già oggetto di attenzione in altre pubblicazioni. Fatta eccezione per due gruppi di ossa ovine, per pochi oggetti in metallo ed in pietra, per qualche peso da telaio fittile e per i coppi già segnalati, gli oggetti rinvenuti sono quasi esclusivamente vasi ceramici (corinzi, attici, greco-orientali e locali) ed offerte coroplastiche (statuette e protomi). La presentazione è organizzata per classi secondo un ordine basato sulla cronologia dei singoli pezzi e non sulla pertinenza alla stratigrafia dello scavo: questa scelta facilita l'esame delle diverse classi di materiali, ma ostacola quello della composizione dei due depositi votivi. Per ogni oggetto una esauriente scheda, oltre alla descrizione, riporta la bibliografia ed un commento. La documentazione grafica e fotografica presentata curata dallo stesso Autore - è accettabile anche se le foto (a colori) dei materiali sono prive di riferimenti metrici e non rispettano un medesimo rapporto di riduzione anche nell'ambito delle stesse tavole. Manca purtroppo una tabella di concordanza fra i numeri di inventario del Museo e quelli del catalogo. L'analisi analitica di tutti i materiali ceramici conservati permette a T.I. di precisare meglio la cronologia del santuario, che va fissata fra metà del VII e metà del VI secolo a.C. (I strato) e fra metà del VI e fine del V secolo a.C. (II strato): viene quindi eliminato lo iato del 550-530 a.C. circa e la fine del santuario può essere messa in rapporto agli eventi del 405 a.C.: si tratta di due acquisizioni certamente importanti. I materiali dello strato I sono quasi 
esclusivamente ceramici, con grande abbondanza di importazioni corinzie (il 51,9\% delle ceramiche): fra le forme del periodo abbondano in particolare balsamari e lucerne (fra cui quella celebre a doppia vasca con protomi umane alternate a protomi d'ariete). La situazione muta sensibilmente nello strato II, in cui diminuisce in modo significativo la presenza di ceramica (da 536 a 105 oggetti: adesso la ceramica più diffusa è quella attica, con il 52,4\%), mentre si registra un notevole aumento delle offerte coroplastiche (dai 16 votivi del I strato si passa ai 70 del II). Quanto a queste ultime ci si limita a rilevare come il carattere cretese delle tre statuette 'gemelle' a matrice (inv. 7497-9, cat. 654-6: p. 168-169, 182, 218-219) sia, come sempre, più asserito che dimostrato ${ }^{6}$.

Alcuni grafici ed una tabella (p. 29-32) aiutano a prendere visione delle differenti composizioni delle ceramiche nelle due diverse fasi ed altri grafici sono presentati per le singole classi ceramiche. Dei grafici con la composizione dell'intero complesso dei materiali dei due strati - e magari anche quelle dei materiali coroplastici - sarebbero stati altrettanto utili. Allo stesso modo la tabella di p. 32, con l'indicazione numerica degli esemplari per singole forme ceramiche divise per funzione e area di produzione non permette di cogliere analogie e differenze fra i due momenti di uso del santuario. T.I. comunque sottolinea questi aspetti nel testo, segnalando anche gli elementi di continuità ravvisabili nei modi deposizionali fra le due fasi, rappresentati dalla presenza di ceramiche legate alle libagioni ed al consumo di bevande in entrambi i periodi.

7 Delle differenze sostanziali vengono peraltro ravvisate fra il tipo dei votivi documentati nel predio Sola e quelli attestati in particolare nel coevo santuario di Bitalemi, l'unico che, grazie a delle evidenze epigrafiche, può con certezza essere interpretato come un Thesmophorion. Queste divergenze permettono a T.I. di mettere convincentemente in dubbio il carattere demetriaco del santuario del predio Sola: piuttosto, la larga attestazione di vasi per la cosmesi nella prima fase e quella di protomi fittili nella seconda suggeriscono la possibilità che il culto fosse tributato ad una divinità femminile e fosse peculiare alla sfera nuziale. Dopo avere discusso la possibile pertinenza ad Hera o ad Artemide e soprattutto ad Afrodite, T.I. propende, nelle pagine conclusive, per Kore, nel suo ruolo di nymphe (p. 227-235). Per il cambiamento documentato negli oggetti dei due diversi strati vengono semplicemente proposti (p. 226-227) un nuovo 'sistema rituale' (che darebbe conto della scomparsa delle lucerne) ed una 'maggiore potenza comunicativa dell'immagine' (che spiegherebbe le protomi). Appare invece condivisibile l'ipotesi che anche nella prima fase esistessero nel santuario delle strutture, magari in materiale deperibile (p. 209): è difficile ammettere che, ad esempio, la lampada a doppia vasca, con anelli di sospensione e foro inferiore per essere inserita su supporto, non trovasse collocazione in un ambiente coperto. L'interesse principale dell'A. è comunque incentrato sul tipo di rituale che aveva luogo nel santuario: non sono quindi presi in considerazione i possibili riflessi che su di esso possono avere avuto le differenti forme di organizzazione politica di Gela.

Da un punto di vista editoriale va lamentata l'assenza di un riassunto in lingua inglese, che avrebbe facilitato la fruizione dell'opera anche per gli studiosi che non hanno particolare dimestichezza con l'italiano. Occorre però forse congratularsi con l'editore per essere riuscito a pubblicare un volume di buona qualità ad un prezzo accettabile, diversamente da quanto si riscontra spesso nell'attuale panorama dell'editoria archeologica in Italia. 
9 Malgrado le perplessità cui si è fatto riferimento, l'opera di T.I. va segnalata senza dubbio come un'interessante acquisizione nell'ambito dell'archeologia della Sicilia antica. Il suo pregio principale è probabilmente quello di avviare una seria riflessione metodologica sugli importanti complessi santuariali portati alla luce a Gela, utilizzando l'intera evidenza archeologica disponibile. Mentre già da tempo una équipe di studio è al lavoro sul santuario di Bitalemi, è da augurarsi che presto vengano riesaminati gli altri complessi cultuali di via Fiume, di Carrubbazza, della Madonna dell'Alemanna, dell'ex scalo ferroviario, entrati da tempo in letteratura grazie alle pronte ed esaustive notizie datene da Orlandini ed Adamesteanu, ma non oggetto di un riesame puntuale ed approfondito come quello condotto sul santuario del predio Sola da Ismaelli. Uno studio sistematico su questi santuari potrebbe contribuire ad acquisire una migliore conoscenza, oltre che della topografia dell'abitato antico, anche delle pratiche cultuali documentate fra VII e V secolo a.C., probabilmente utile in una prospettiva non solo geloa. Forse è fra i resti del suo straordinario passato che può trovarsi un futuro migliore per Gela, accantonati i miraggi di rovinose speculazioni.

\section{NOTE}

1. Cfr. P. ORSI, «Gela. Scavi del 1900-1905», MonAL 16 (1906), coll. 24-26; M. VICKERS, «'... at Terranova one gets more for one's money than at Rome': Arthur and Margaret Evans in Gela, 1887-1896», in R. PANVINI, F. GIUDICE (edd.), Ta Attikà. Veder greco a Gela. Ceramiche attiche figurate dall'antica colonia, Roma, 2003, p. 239-242.

2. Vd. E. HYTTEN, M. MARCHIONI, Industrializzazione senza sviluppo: Gela, una storia meridionale, Milano, 1970; più di recente P. SAITTA, L. PELLIZzonI, «Lo chiamavano 'sviluppo': il complicato rapporto di Gela con l'ENI», Archivio di studi urbani e regionali 96 (2009), p. 158-188 con bibliografia.

3. P. ORLANDINI, «Gela. La stipe votiva arcaica del predio Sola», MonAL 46 (1963), col. 1-78.

4. Ibid., c. 4: «Lo scavo ... cadeva ... là dove al ripido pendio iniziale segue un breve tratto pianeggiante.»

5. Ibid., c. 76. Per l'impiego del termine cf. E. LIPPOLIS, G. Rocco, Archeologia greca. Cultura, società, politica e produzione, Milano, 2011, p. 95-99.

6. Per una recente trattazione degli esemplari cretesi, O. PILZ, Frühe matrizengeformte Terrakotten auf Kreta. Votivpraxis und Gesellschaftsstruktur in spätgeometrischer und früharchaischer Zeit, Möhnesee, 2011.

\section{AUTORI}

\section{NICOLA CUCUZZA}

Università degli studi di Genova 